\title{
Short stump and high anastomosis pull-through (SHiP) procedure for delayed coloanal anastomosis with no protective stoma for low rectal cancer
}

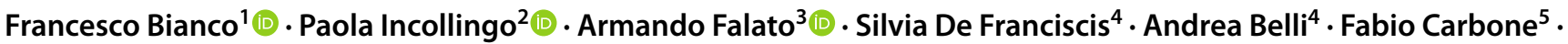 \\ Gaetano Gallo ${ }^{6}$. Mario Fusco $^{7} \cdot$ Giovanni Maria Romano $^{8}$
}

Received: 25 November 2020 / Accepted: 2 March 2021 / Published online: 16 March 2021

(c) The Author(s) 2021

\begin{abstract}
Despite advances in coloanal anastomosis techniques, satisfactory procedures completed without complications remain lacking. We investigated the effectiveness of our recently developed 'Short stump and High anastomosis Pull-through' (SHiP) procedure for delayed coloanal anastomosis without a stoma. In this retrospective study, we analysed functional outcomes, morbidity, and mortality rates and local recurrence of 37 patients treated using SHiP procedure, out of the 282 patients affected by rectal cancer treated in our institution between 2012 and 2020. The inclusion criterion was that the rectal cancer be located within $4 \mathrm{~cm}$ from the anal margin. One patient died of local and pulmonary recurrence after 6 years, one developed lung and liver metastases after 2 years, and one experienced local recurrence 2.5 years after surgery. No major leak, retraction, or ischaemia of the colonic stump occurred; the perioperative mortality rate was zero. Five patients (13.51\%) had early complications. Stenosis of the anastomosis, which occurred in nine patients (24.3\%), was the only long-term complication; only three (8.1\%) were symptomatic and were treated with endoscopic dilation. The mean Wexner scores at 24 and 36 months were 8.3 and 8.1 points, respectively. At the 36-month check-up, six patients (24\%) had major LARS, ten (40\%) had minor LARS, and nine (36\%) had no LARS. The functional results in terms of LARS were similar to those previously reported after immediate coloanal anastomosis with protective stoma. The SHiP procedure resulted in a drastic reduction in major complications, and none of the patients had a stoma.
\end{abstract}

Keywords Pull- through $\cdot$ Coloanal anastomosis $\cdot$ Rectal cancer $\cdot$ Stoma $\cdot$ Turnbull-Cutait

Francesco Bianco

bianco.bar@tin.it

1 General Surgery Unit, San Leonardo Hospital, Castellammare di Stabia, ASL NA3 Sud, Viale Europa, 283, 80053 Naples, Italy

2 General Surgery Unit, Department of Advanced Biomedical Sciences, University Federico II, San Leonardo Hospital, Castellammare di Stabia, ASL NA3 Sud, Naples, Italy

3 General and Laparoscopic Surgery Unit, San Giuliano Hospital, Giugliano in Campania, Naples, Italy

4 Istituto Nazionale Tumori IRCCS Fondazione G. Pascale, Naples, Italy

5 Department of Advanced Biomedical Sciences, University Federico II, Naples, Italy

6 Department of Medical and Surgical Sciences, University of Catanzaro, Catanzaro, Italy

7 Cancer Registry Unit, ASL NA3 Sud, Torre del Greco, Italy

8 San Michele Hospital, Maddaloni, Italy

\section{Introduction}

Coloanal anastomosis remains the subject of lively clinical debate despite modern advances in the technique, given that outcomes remain unsatisfactory [1-4]. Since Rullier et al. published their criteria in 2013, there has been a notable increase in the rate of surgeries that conserve the pelvic floor, thereby promoting the possibility of expanding this type of reconstruction to all patients who have no invasion of the pelvic floor muscles [5]. Nevertheless, none of the coloanal anastomosis procedures used to date is without notable complications; they usually do not provide satisfactory functional outcomes [6, 7].

In this study, we investigated the effectiveness of our recently developed 'Short stump and High anastomosis Pullthrough' (SHiP) procedure for delayed coloanal anastomosis without a stoma. This method represents a modification of the previously described pull-through procedure [8-11] 
that attempts to address the aforementioned limitations. The development of this technique dates back to 2012 as reported in previous studies [12-14]; we refined this procedure based on our experience with 37 consecutive patients via continuous modifications.

In this retrospective study, we analysed the functional outcomes as well as the morbidity and mortality rates of 37 patients treated for low rectal cancer using the SHiP procedure out of the 282 patients affected by rectal cancer treated in our institution between 2012 and 2020.

\section{Materials and methods}

This was a retrospective single-centre study and is reported according to the Strengthening the Reporting of Observational Studies in Epidemiology (STROBE) statement for cohort studies [15].

The ethics committee of our institution approved this study. Between 2012 and 2020, 37 patients were treated for low rectal cancer using the SHiP procedure out of the 282 patients affected by rectal cancer.

The inclusion criterion were as follows: rectal cancer be located within $4 \mathrm{~cm}$ from the anal margin, no radiological sign of cancer invasion of the internal sphincter and/or levator ani muscle, strong motivation of the patient to avoid temporary ileostomy. All patients were informed of alternative surgical treatments and provided written informed consent regarding the procedure and treatment of personal data.
The surgical technique carried out is a conventional low anterior resection, including high vascular ligation, complete left colon, splenic flexure and half transverse colon mobilization (up to middle colic vessels) and total mesorectal excision. Anal mucosectomy and transanal rectal section are then performed and the left colonic stump is pulled through the anus. Four referral stitches (which will be the markers for fashioning the delayed anastomosis) are placed between the colic serosa and the upper verge of the anal canal (cranially to the internal sphincter). In the second stage of the procedure the adhesions between the colonic stump and the anal canal are bluntly dissected until the marker stitches. The colonic stump is then sectioned at this level, leaving the anal canal free from the residual colon, and a "high" anastomosis completed with four additional stitches [12, 14]. In order to minimize the discomfort between the two steps of the procedure, in the second half of the study, the length of the stump was reduced and a short stump of approximately $2 \mathrm{~cm}$-length from the anocutaneous line was performed (Fig. 1). Thirtyseven patients ( 25 men and 12 women) aged between 38 and 74 years underwent the procedure; preoperative patient data (American Society of Anesthesiologists score, body mass index, and comorbidities) are shown in Table 1. Any cardiovascular and respiratory disease, obesity, kidney failure, diabetes and cerebral ischaemia were considered as comorbidities. Two patients had undergone previous rectoanal surgeries for benign disease (hemorrhoidectomy and transanal fistula excision), one had undergone a right hemicolectomy for cancer, and two previously underwent local transanal adenocarcinoma excision that required radical
Fig. 1 Second stage: resection of the colic stump and fashioning of the high coloanal anastomosis; a stump at the end of the first stage; $\mathbf{b}$ identification of the referral stitches, adhesiolysis, section of the stump and completion of the High coloanal anastomosis; $\mathbf{c}$ high coloanal anastomosis at the end of the second stage of the procedure
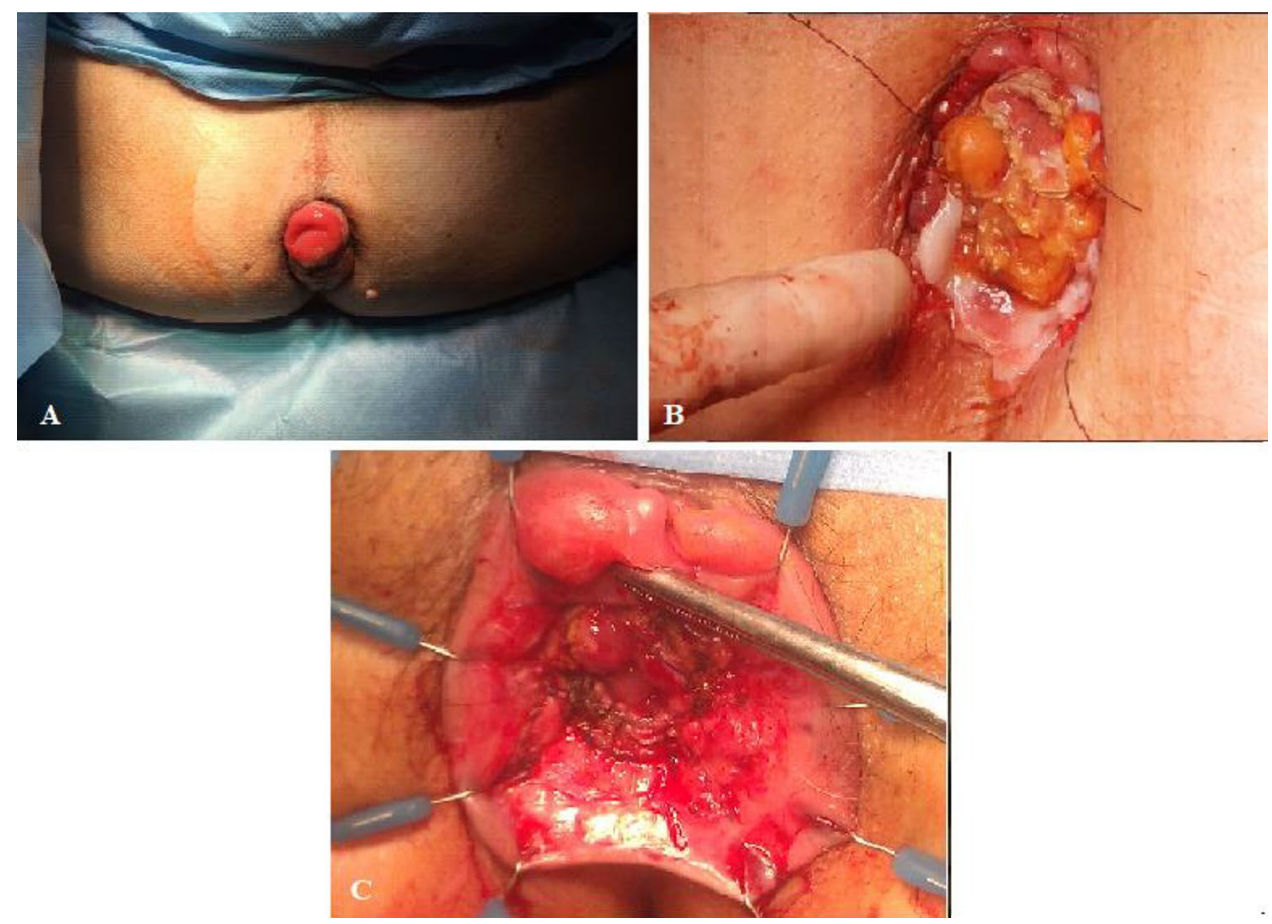
Table 1 Patients' anagraphic data

\begin{tabular}{llll}
\hline Age at diagnosis (mean) & $60.8( \pm 8.82)$ & & \\
BMI (mean) & $25.7( \pm 3.74)$ & & \\
Sex (m/f) & $25 / 12$ & & \\
Preop CRT (y/n) & $32 / 5$ & & III \\
ASA (no.) & I & II & 12 \\
& 2 & 23 & High \\
Comorbidity (no.) & None (0) & Low (1) & mor $)$ \\
& & & 7 \\
\hline
\end{tabular}

surgery. Thirty-two patients (86.5\%) were administered neoadjuvant therapy before surgery (30 and two with long- and short-course chemoradiotherapy, respectively). All patients were instructed not to sleep in the supine position or to sit without dedicated supports during the period between the two surgical steps to avoid damage to the colonic stump. Patients returned for follow-up visits $1,3,6,12,18$, and 24 months after surgery and every year thereafter.

At each follow-up, patients underwent rectal digital exploration and the Cleveland Clinic Incontinence Score (Wexner score) and low anterior resection syndrome (LARS) score were used to evaluate Anal continence and function [16-19].

Two patients had an average follow-up of less than 1 year and were, therefore, not included in the statistical analyses of the Wexner and LARS scores.

\section{Results}

The mean age of the patients was 60.8 years (range 38-74). The mean BMI was $25.7 \pm 3.74$ (range 18-38). Perioperative data are summarized in Table 2. Histological results after resection are reported in Table 3. Considering patient's comorbidities, BMI, ASA score, previous abdominal surgery, tumor size, and hostile anatomy, laparotomy was performed in $23(62 \%)$ patients while 11 (30\%) underwent a laparoscopic approach and $3(8 \%)$ a robotic approach (Table 2). The second surgical step (resection of the transanal colonic stump) was performed an average of $13.8 \pm 4.3$ days later; 22

Table 2 Surgical data

\begin{tabular}{llll}
\hline Surgical tech (no.) & Open & Laparoscopic & Robotic \\
& 23 & 11 & 3 \\
Distance from anal verge & $4 \mathrm{~cm}$ & $3 \mathrm{~cm}$ & $2 \mathrm{~cm}$ \\
$\quad$ (no.) & 23 & 9 & 5 \\
$\begin{array}{l}\text { Interval between the two sur- } \\
\text { gical steps (days mean) }\end{array}$ & $13.8( \pm 4.28)$ & \\
Ileostomy (no.) & 0 & & \\
\hline
\end{tabular}

Table 3 Postoperative data and complications

\begin{tabular}{ll}
\hline Procedure failure & None \\
Perioperative mortality & None \\
Stump retraction/ischaemia & None \\
Coloanal leak & None \\
30 days compl sec. Dindo (no.) & \\
I & 1 \\
II & 2 \\
III A & 1 \\
III B & 1 \\
Long-term complications & Stenosis = 9 \\
Stage (AJCC vs.7) (no.) & \\
Stage 0 & 6 \\
Stage I & 27 \\
Stage II A & 2 \\
Stage III A & 1 \\
Stage III B & 1 \\
\hline
\end{tabular}

patients were discharged between the 5 th and 7 th postoperative days and were then readmitted for 2 days to undergo the second step. As such, the mean total length of hospital stay was 8.2 days. Some of the first 18 patients experienced anastomotic stenosis; therefore, anal dilatators were routinely used in the first 6 months after surgery for all successive patients (Table 3). Furthermore, since no stump retraction was observed in the first half of the study (i.e., the first 18 patients), a short stump of approximately $2 \mathrm{~cm}$ length from the anocutaneous line was performed in all subsequent patients to reduce discomfort between the two steps of surgery (Fig. 1).

One patient died of local and pulmonary recurrence after 6 years, another developed lung and liver metastases after 2 years, and a third experienced local recurrence 2.5 years after surgery. None of the patients experienced failure of the surgical technique (Table 3 ). There were no incidences of major leaks, retractions, or ischaemia in the colonic stump; moreover, no perioperative fatalities occurred. Five patients (13.51\%) had early complications within 30 days of surgery: three patients (one and two with Clavien-Dindo grades of I and II, respectively) were treated conservatively with medical therapy, another underwent relaparotomy for median suture dehiscence (Clavien-Dindo IIIB), and the fifth underwent computed tomography-guided percutaneous drainage of a pelvic abscess (Clavien-Dindo IIIA) [20]. Stenosis of the anastomosis was the only long-term complication observed. Stenosis on physical examination or colonoscopy was found in nine patients $(24.3 \%)$; of these, only three $(8.1 \%)$ were symptomatic and were treated with endoscopic dilatations, while the remaining six were asymptomatic and were treated with anal dilators. Following the routine introduction of anal dilators, only three patients developed 
anastomotic stenosis owing to their non-compliance regarding the use of prescribed dilators. The postoperative complications are listed in Table 3. Two patients were prescribed anti-diarrheal drugs 6 months after surgery.

The mean Wexner scores at 12, 24, and 36 months were $10.2,8.3$, and 8.1, respectively. The 57 and $30 \%$ of the patients had major LARS at 12 and 24 months, respectively. At the 36-month check-up, six patients (24\%) had a major LARS, 10 (40\%) had minor LARS, and nine (36\%) had no LARS. The scores are fully reported in Table 4 (Figs. 2, 3).

\section{Discussion}

Rullier et al. indicated that it is possible to reconstruct pelvic floor muscles with full anatomic integrity if they are free from neoplasia via sphincter-saving coloanal anastomosis [5]. Thus, it is possible to achieve such reconstruction while abolishing the need for a few centimeters' of free margin; however, the ideal ultra-low coloanal anastomosis method (hand-made or stapled) remains the subject of debate [1-3].

To date, a high proportion of patients experience postoperative complications due to anastomotic leaks of coloanal anastomoses or to secondary events following reversal surgery for the closure of protective ileostomies [21-25]. Therefore, it is necessary to identify anastomosis techniques that can greatly reduce the rate of postoperative complications, which currently occur in up to $40 \%$ of patients with coloanal anastomoses and can delay or even prevent patients' access to adjuvant chemotherapy and risk forming a definitive stoma [22, 26, 27]. As a consequence, patients with ultra-low coloanal anastomoses still require a temporary derivative stoma [28] which significantly affects their quality of life (particularly during chemoradiotherapy) [29].

Owing to such considerations, the impetus for this study was the need for a technique that would create the anastomosis only after the transposed colon had attached to the plane of the pelvic muscles, without risking leakage. These considerations were also the bases for the development of

Table 4 Functional results expressed by the means of the Wexner score and LARS score (Low Anterior Resection Syndrome score)

\begin{tabular}{lccr}
\hline & \multicolumn{1}{l}{$12 \mathrm{M}$} & \multicolumn{1}{c}{$24 \mathrm{M}$} & \multicolumn{1}{c}{$36 \mathrm{M}$} \\
\hline WEXNER (mean) & $10.2( \pm 3.9)$ & $8.3( \pm 4.7)$ & $8.1( \pm 4.8)$ \\
LARS (mean) & $31.1( \pm 4.9)$ & $24.8( \pm 8.2)$ & $23.2( \pm 9.3)$ \\
MAJOR LARS (no.) & $20(57 \%)$ & $9(30 \%)$ & $6(24 \%)$ \\
MINOR LARS (no.) & $15(43 \%)$ & $15(50 \%)$ & $10(40 \%)$ \\
NO LARS (no.) & $0(0 \%)$ & $6(20 \%)$ & $9(36 \%)$ \\
\hline
\end{tabular}

No $L A R S=$ from 0 to 20 , minor $\mathrm{LARS}=$ from 21 to 29 , major LARS $=$ from 30 to 42

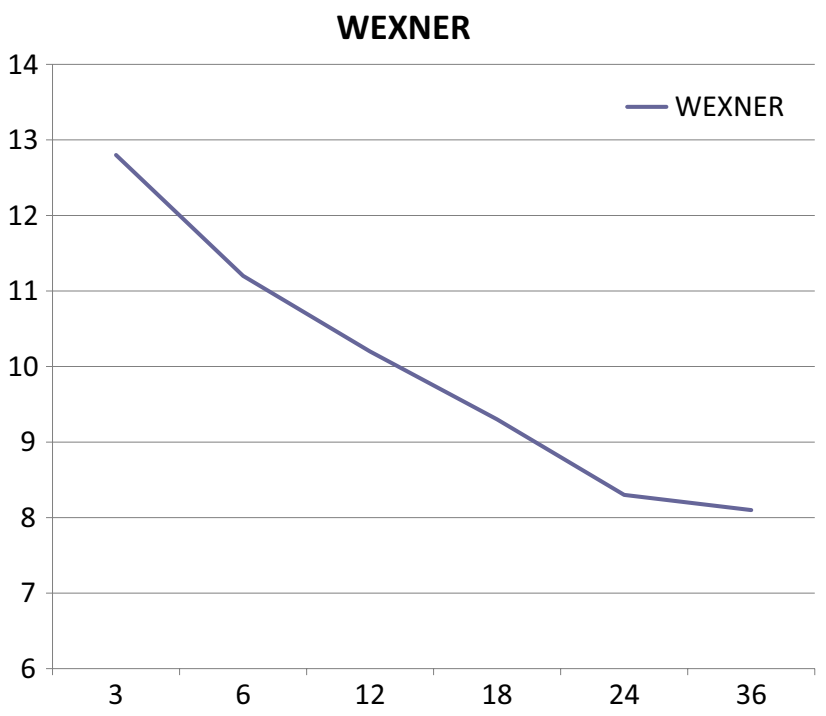

Fig. 2 Wexner score trend over time

the pull-through technique $[8,9]$, which was abandoned in the 1980s following the introduction of mechanical staplers.

In the previously used pull-through technique, the colonic stump was resected after a period varying from 7 to 14 days, giving time to the serosa to adhere to the anal canal muscles (delayed coloanal anastomosis) and prohibiting the detachment of these adhesions in the second surgical step [11]. The latter is the main technical distinction from the modified technique that we have now adopted. In the SHiP procedure the adhesions are partially detached up to the landmark stitches allowing a high proximal stump resection and anastomosis, as described in our previous studies [12, 14].

The old pull-though procedure had some drawbacks that led to its gradual abandonment. The first limitation was sphincter incontinence due to the presence of a residual colonic stump inside the anal canal, which impeded the correct contraction of the muscles. That was a consequence of

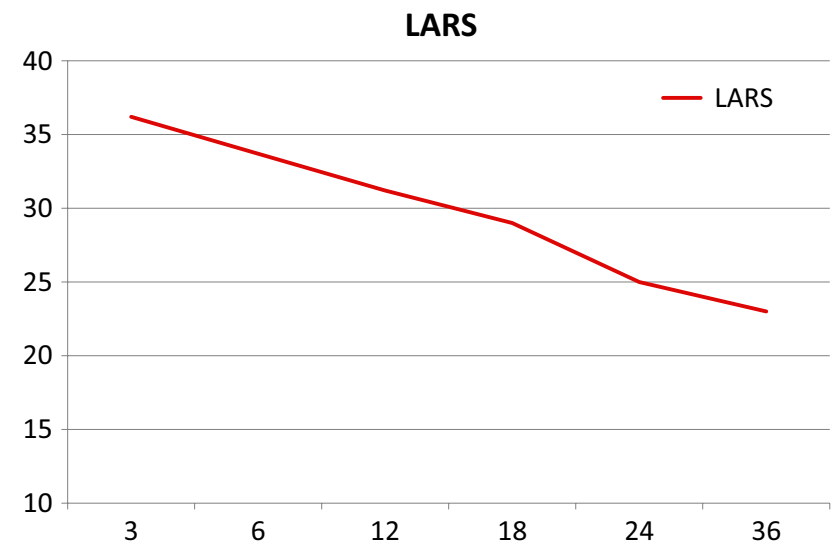

Fig. 3 Low anterior resection syndrome score trend over time 
the technical prohibition against detaching the adhesions, which frequently led in the old procedure to a very low resection of stump very close to anocutaneous margin. In both the old procedure as well as the technique more recently described by French authors in 2011, it was forbidden to sever the adhesions inside the anal canal to avoid the risk of entering the pelvic cavity by detaching the colonic stump. This has led to the presence of a colonic remnant in the anal canal that affected sphincter contraction and continence [11, 30].

The second drawback of the old procedure was the risk of anastomotic leak [10,31-33] and of ischaemia in the colonic stump transposed in the anal canal, the so-called "guillotine effect". This was owing to the discrepancy between the tightening of the anal canal muscles and the mesocolonic diameters, as well as to terminal vascularization at the sigmoid level [12, 31].

The third drawback was the occurrence of stenosis in the coloanal anastomosis after the second step of the colonic stump resection [31].

Finally, the fourth drawback was patient discomfort during the 7- to 14-day period between the first and second steps of the procedure caused by the encumbrance of the colonic stump itself.

Our experience with 37 consecutive patients permitted, through "continuous modifications", the development of our modified SHiP procedure for delayed coloanal anastomosis without a protective stoma; this overcame all the drawbacks of the old procedure (Fig. 4).

As described in our previous papers [12-14] the technical modifications that were hallmarks of the new procedure included the placement of very proximal suture "markers" in the anal canal during the first step of the procedure. This allowed creating a coloanal anastomosis that was very high and proximal to the anal canal in the second step, thereby freeing the anal canal from the residual colonic stump and allowing better sphincter activity. Additionally, the complete mobilization of the left colon up to the middle colonic vessels allowed for transposition of the left colon in the anal canal, whose vascularization (in contrast to that of the sigmoid colon) is arched and not terminal. This latter and the minor diameter discrepancy with the anal canal of the left mesocolon as compared to the meso-sigmoid prevented ischaemia of the colonic stump. This extended mobilization also allows the left colon to lie floppily and tension-free in the pelvis along the sacral curve, thus avoiding any retraction of colonic stump [12, 14].

These modifications made it possible to avoid the risk both of anastomotic leaks and colonic stump ischaemia, thereby making a protective stoma unnecessary. The favourable results obtained after our initial experiences made it possible to realize that it was not necessary to create an overly long colonic stump as required by the old technique; instead, a stump of a few centimeters (similar to the size of a mucosal rectal prolapse) is sufficient (Fig. 1).

Shortening the colonic stump prevents considerable discomfort caused by the stump itself between the two steps of the procedure. The operations were performed as open, laparoscopic, and robotic surgeries; hence, it was possible to avoid scarring with no abdominal incision while the specimen was extracted through the anus. Patients with bulky tumors were excluded from the non-scarring procedure, as extraction through the anus would cause excessive stretching and damage of the sphincter; in such cases, the specimens were extracted through an abdominal incision.

None of the 37 patients who underwent surgery with the modified technique experienced postoperative mortality or anastomotic leaks, and only one patient underwent relaparotomy for median suture dehiscence. Moreover, only two patients experienced local recurrence, and one died of metastases.

Stenosis of the anastomosis occurred in nine patients, among whom three were treated with endoscopic dilation and the remaining six utilized anal dilators as outpatients. This experience prompted us to use anal dilators starting from 30 days up to the 6th month after surgery (until the anastomosis was stable); this made it possible to eliminate stenosis in all patients and to improve functional results.
Fig. 4 Schematic representation of the differences between the old and the new pull-through technique

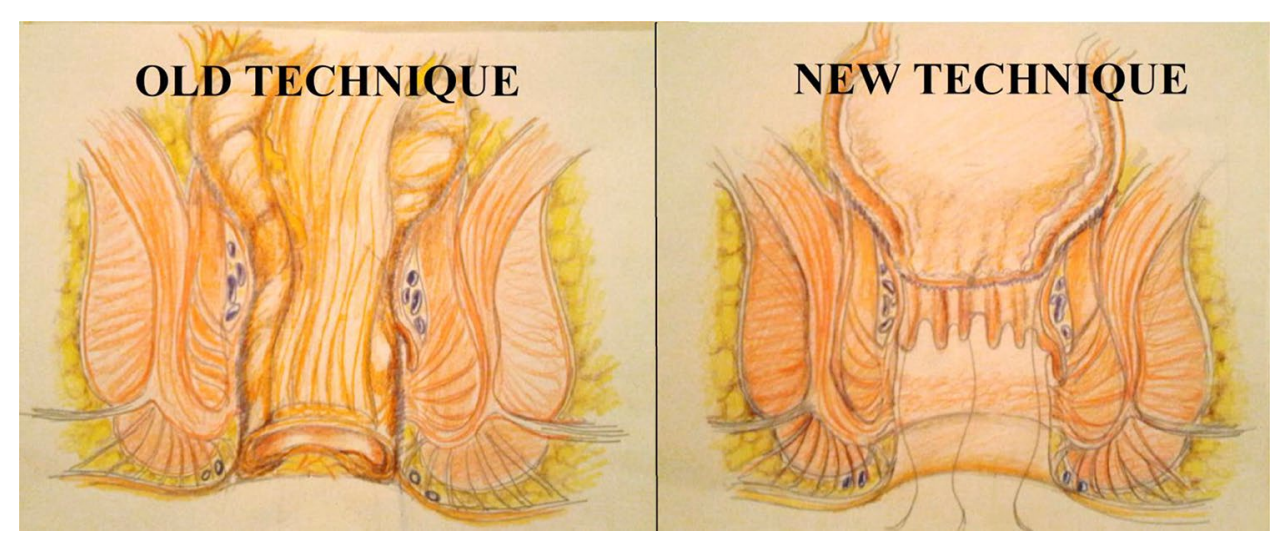


As previously reported, foecal incontinence and LARS progressively improve during the 2 years after surgery in patients with immediate coloanal anastomosis; there are no differences in functional outcomes between patients who undergo straight (end-to-end) or lateral (side-toend) anastomosis and those who undergo reconstruction via the J-pouch procedure after 1 year has passed [34]. Even in our experience, patients undergoing the SHiP procedure had progressive improvement in continence and LARS up to 3 years post-surgery (Table 4; Figs. 2, 3).

The functional results obtained using the $\mathrm{SHiP}$ as measured using the LARS and Wexner scores were at least similar to those reported in the literature after immediate coloanal anastomosis, but without the need for any protective ileostomy [30, 34-37]. Compared to immediate coloanal anastomosis, the SHiP procedure allowed for a drastic reduction in major complications despite the fact that none of the patients had a definitive stoma. Worse functional results were achieved in patients with high body mass indices, in the one who had a previous right hemicolectomy, and women with more natural childbirths. This suggested a need for more careful selection of patients.

Previously published data from patients who underwent immediate coloanal anastomosis with a protective stoma reveal that $20-40 \%$ of them had major complications with temporary protective stomas becoming definitive [22-24] as well as a proportion that developed metabolic complications [38]. Furthermore, favourable functional outcome rates were inversely proportional to postoperative complication rates related to the coloanal anastomosis. As such, the marked reduction in complications when using the SHiP procedure reflects in an improvement in long-term functional results.

The greatest improvement is undoubtedly in terms of patient satisfaction, as subjects will not require a stoma (even for a limited period of time) nor will they require another intra-abdominal operation for stoma reversal.

The aspects described above have an important impact on deciding whether to perform SHiP or an immediate coloanal anastomosis with protective ileostomy. It is important to highlight that some patients will undergo postoperative adjuvant chemotherapy; therefore, the occurrence of complications due to the first and/or reversal surgery might force the patient to undergo chemotherapy with a stoma, postponing its closure and thus markedly worsening the patient's quality of life $[39,40]$.

Our study has some limitations. It is a single-centre study with lack of a control group. Although the data were collected prospectively, data analysis was performed retrospectively. Further randomized trials will be needed [41].

\section{Conclusion}

The functional outcomes obtained using the SHiP procedure were at least on par with those reported in the literature after immediate coloanal anastomosis in terms of LARS. Indeed, the SHiP procedure resulted in a drastic reduction in major complications, and none of the patients required a stoma. Results from multicenter randomized controlled trials are now warranted. Nevertheless, our experience over 8 years of practice, and the observed outcomes both in terms of functional results and complications, indicate that the SHiP procedure might play a fundamental role in the treatment of ultra-low rectal cancer.

Author contributions All the above listed Authors have given substantial contributions to conception and design; acquisition of data, analysis and interpretation of data; drafting the article or revising it critically for important intellectual content; and final approval of the version to be published.

Funding No funding was received for conducting this study.

Data availability All data generated or analysed during this study are included in this published article.

\section{Declarations}

Conflict of interest All authors certify that they have no affiliations with or involvement in any organization or entity with any financial interest or non-financial interest in the subject matter or materials discussed in this manuscript.

Ethics approval The ethics committee of our institution approved this study.

Consent for publication All patients were informed of alternative surgical treatments and provided written informed consent regarding the procedure and treatment of personal data.

Open Access This article is licensed under a Creative Commons Attribution 4.0 International License, which permits use, sharing, adaptation, distribution and reproduction in any medium or format, as long as you give appropriate credit to the original author(s) and the source, provide a link to the Creative Commons licence, and indicate if changes were made. The images or other third party material in this article are included in the article's Creative Commons licence, unless indicated otherwise in a credit line to the material. If material is not included in the article's Creative Commons licence and your intended use is not permitted by statutory regulation or exceeds the permitted use, you will need to obtain permission directly from the copyright holder. To view a copy of this licence, visit http://creativecommons.org/licenses/by/4.0/.

\section{References}

1. Ramage L, Mclean P, Simillis C, Qiu S, Kontovounisios C, Tan E, Tekkis P (2018) Functional outcomes with handsewn versus 
stapled anastomoses in the treatment of ultralow rectal cancer. Updates Surg 70(1):15-21

2. Huttner FJ, Tenckoff S, Jense K et al (2015) Meta-analysis of reconstruction technique after low anterior resection for rectal cancer. Br J Surg 102:735-745

3. Shaun Brown DO, Margolin DA, Altom LK et al (2018) Morbidity following coloanal anastomosis: a comparison of colonic J-pouch vs straight anastomosis. Dis Colon Rectum 61:156-161

4. Heriot AG, Tekkis PP, Constantinides V et al (2006) Meta-analysis of colonic reservoirs versus straight coloanal anastomosis after anterior resection. Br J Surg 93:19-32

5. Rullier E, Denost Q, Vendrely V, Rullier A, Laurent C (2013) Low rectal cancer: classification and standardization of surgery. Dis Colon Rectum 56(5):560-567

6. Garg PK, Goel A, Sharma S, Chishi N, Gaur MK (2019) Protective diversion stoma in low anterior resection for rectal cancer: a metaanalysis of randomized controlled trials. Visc Med 35(3):156-160

7. Pisarska M, Gajewska N, Małczak P et al (2018) Defunctioning ileostomy reduces leakage rate in rectal cancer surgery-systematic review and meta-analysis. Oncotarget 9(29):20816-20825

8. Turnbull RB, Cuthbertson A (1961) Abdominorectal pull-through resection for cancer and for Hirschsprung's disease. Delayed posterior colorectal anastomosis. Cleve Clin Q 28:109-115

9. Cutait DE, Figliolini FJ (1961) A new method of colorectal anastomosis in abdominoperineal resection. Dis Colon Rectum 4(5):335-342

10. Remzi FH, Gazzaz E, Kiran RP, Kirat HT, Fazio VW (2009) Outcomes following Turnbull-Cutait abdominoperineal pull-through compared with coloanal anastomosis. Br J Surg 96(4):424-429

11. Jarry J, Faucheron JL, Moreno W, Bellera CA, Evrard S (2011) Delayed colo-anal anastomosis is an alternative to prophylactic diverting stoma after total mesorectal excision for middle and low rectal carcinomas. Eur J Surg Oncol 37(2):127-133

12. Bianco F, De Franciscis S, Belli A, Ragone V, Romano GM (2015) A pull-through delayed "high" coloanal anastomosis: new tricks to refresh an old procedure. Tech Coloproctol 19(4):259-261

13. Bianco F, Belli A, De Franciscis S, Falato A, Romano GM (2016) "Scarless" and no-stoma surgery for low rectal cancer: the laparoscopic pull-through delayed "high" colo-anal anastomosis. Updates Surg 68(1):99-104

14. Bianco F, Falato A, Belli A, De Franciscis S, De Leon Valdez JD, Romano GM (2017) Modified pull-through technique with a delayed high coloanal anastomosis: no stoma and scarless surgery for low rectal cancer. Dis Colon Rectum 60(10):1113

15. Von Elm E, Altman DG, Egger M et al (2014) The strengthening the reporting of observational studies in epidemiology (STROBE) statement: guidelines for reporting observational studies. Int $\mathrm{J}$ Surg 12:1495-1499

16. Jorge JMN, Wexner SD (1993) Etiology and management of fecal incontinence. Dis Colon Rectum 36(1):77-97

17. Emmertsen KJ, Laurberg S (2012) Low anterior resection syndrome score: development and validation of a symptom-based scoring system for bowel dysfunction after low anterior resection for rectal cancer. Ann Surg 255(5):922-928

18. Croese AD, Lonie JM, Trollope AF et al (2018) A meta-analysis of the prevalence of low anterior resection syndrome and systematic review of risk factors. Int J Surg 56:234-241

19. Juul T, Elfeki H, Christensen $P$ et al (2019) Normative data for low anterior resection syndrome score (LARS score). Ann Surg 269(6):1124-1128

20. Dindo D, Demartines N, Clavien P-AA (2004) Classification of surgical complications: a new proposal with evaluation in a cohort of 6336 patients and results of a survey. Ann Surg 240(2):205-213

21. Rahbari NN, Weitz J, Hohenberger W et al (2010) Definition and grading of anastomotic leakage following anterior resection of the rectum: a proposal by the International study group of rectal cancer. Surgery 147(3):339-351

22. Musters GD, Atema JJ, van Westreenen HL, Buskens CJ, Bemelman WA, Tanis PJ (2016) Ileostomy closure by colorectal surgeons results in less major morbidity: results from an institutional change in practice and awareness. Int $\mathrm{J}$ Colorectal Dis 31(3):661-667

23. Man VCM, Choi HK, Law WL, Foo DCC (2016) Morbidities after closure of ileostomy: analysis of risk factors. Int J Colorectal Dis 31(1):51-57

24. Sharma A, Deeb AP, Rickles AS, Iannuzzi JC, Monson JRT, Fleming FJ (2013) Closure of defunctioning loop ileostomy is associated with considerable morbidity. Colorectal Dis 15(4):458-462

25. Chan DKH, Ng J, Koh FH et al (2019) Journey for patients following ileostomy creation is not straightforward. Int J Colorectal Dis 34(12):2075-2080

26. David GG, Slavin JP, Willmott S, Corless DJ, Khan AU, Selvasekar CR (2010) Loop ileostomy following anterior resection: is it really temporary? Colorectal Dis 12(5):428-432

27. Celerier B, Denost Q, Van Geluwe B, Pontallier A, Rullier E (2016) The risk of definitive stoma formation at 10 years after low and ultralow anterior resection for rectal cancer. Colorectal Dis 18(1):59-66

28. Chude GG, Rayade NV, Patris V et al (2008) Defunctioning loop ileostomy with low anterior resection for distal rectal cancer: should we make an ileostomy as a routine procedure? A prospective randomized study. Hepatogastroenterology 55(86-87):1562-1567

29. Hajibandeh S, Hajibandeh S, Sarma DR et al (2019) Meta-analysis of temporary loop ileostomy closure during or after adjuvant chemotherapy following rectal cancer resection: the dilemma remains. Int J Colorect Dis 34(7):1151-1159

30. Barugola G, Bertocchi E, Ca S et al (2018) Hostile pelvis: how to avoid permanent stoma. Updates Surg 70:459-465

31. Hallet J, Milot H, Drolet S, Desrosiers E, Grégoire RC, Bouchard A (2014) The clinical results of the Turnbull-Cutait delayed coloanal anastomosis: a systematic review. Tech Coloproctol 18(6):579-590

32. Sage P-Y, Trilling B, Waroquet P-A, Voirin D, Girard E, Faucheron J-L (2018) Laparoscopic delayed coloanal anastomosis without diverting ileostomy for low rectal cancer surgery: 85 consecutive patients from a single institution. Tech Coloproctol 22(7):511-518

33. Biondo S, Trenti L, Espín E et al (2012) Post-surgical complications and mortality after two-stage coloanal anastomosis using the Turnbull-Cutait procedure. Cir Esp 90(4):248-253

34. Pucciarelli S, Del Bianco P, Pace U et al (2019) Multicentre randomized clinical trial of colonic J-pouch or straight stapled colorectal reconstruction after low anterior resection for rectal cancer. Br J Surg 106(9):1147-1155

35. Trenti L, Galvez A, Biondo S et al (2018) Quality of life and anterior resection syndrome after surgery for mid to low rectal cancer: a cross-sectional study. Eur J Surg Oncol 44(7):1031-1039

36. Digennaro R, Tondo M, Cuccia F et al (2013) Coloanal anastomosis or abdominoperineal resection for very low rectal cancer: what will benefit, the surgeon's pride or the patient's quality of life ? Int J Colorectal Dis 28:949-957

37. Bondeven P, Emmertsen KJ, Laurberg S et al (2015) Neoadjuvant therapy abolishes the functional benefits of a larger remnant, as measured by magnetic resonance imaging after restorative rectal cancer surgery. Eur J Surg Oncol 41(11):1493-1499

38. Murken DR, Bleier JIS (2019) Ostomy-related complications. Clin Colon Rectal Surg 32(3):176-182

39. Ayaz-Alkaya S (2019) Overview of psychosocial problems in individuals with stoma: a review of literature. Int Wound $\mathbf{J}$ 16(1):243-249 
40. Nugent KP, Daniels P, Stewart B, Patankar R, Johnson CD (1999) Quality of life in stoma patients. Dis Colon Rectum 42(12):1569-1574

41. Biondo S, Trenti L, Galvez A et al (2017) Two-stage TurnbullCutait pull-through coloanal anastomosis versus coloanal anastomosis with protective loop ileostomy for low rectal cancer. Protocol for a randomized controlled trial (Turnbull-BCN). Int J Colorectal Dis 32(9):1357-1362
Publisher's Note Springer Nature remains neutral with regard to jurisdictional claims in published maps and institutional affiliations. 